\title{
TOPOLOGICAL ENTROPY FOR THE CANONICAL COMPLETELY POSITIVE MAPS ON GRAPH $C^{*}$-ALGEBRAS
}

\author{
Ja A. Jeong and Gi hyun Park
}

Let $C^{*}(E)=C^{*}\left(s_{e}, p_{v}\right)$ be the graph $C^{*}$-algebra of a directed graph $E=\left(E^{0}, E^{1}\right)$ with the vertices $E^{0}$ and the edges $E^{1}$. We prove that if $E$ is a finite graph (possibly with sinks) and $\phi_{E}: C^{*}(E) \rightarrow C^{*}(E)$ is the canonical completely positive map defined by

$$
\phi_{E}(x)=\sum_{e \in E^{1}} s_{e} x s_{e}^{*},
$$

then Voiculescu's topological entropy ht $\left(\phi_{E}\right)$ of $\phi_{E}$ is $\log r\left(A_{E}\right)$, where $r\left(A_{E}\right)$ is the spectral radius of the edge matrix $A_{E}$ of $E$. This extends the same result known for finite graphs with no sinks. We also consider the map $\phi_{E}$ when $E$ is a locally finite irreducible infinite graph and prove that $\sup _{E^{\prime}}\left\{\operatorname{ht}\left(\phi_{E^{\prime}}\right)\right\} \leqslant \operatorname{ht}\left(\phi_{E}\right)$, where the supremum is taken over the set of all finite subgraphs of $E$.

\section{INTRODUCTION}

Given a directed graph $E$ with the vertex set $E^{0}$ and the edge set $E^{1}$ it is well known that there exists a universal $C^{*}$-algebra $C^{*}(E)$ generated by partial isometries $\left\{s_{e} \mid e \in E^{1}\right\}$ and mutually orthogonal projections $\left\{p_{v} \mid v \in E^{0}\right\}$ satisfying certain relations determined by the graph $E$. A classical Cuntz-Krieger algebra $\mathcal{O}_{A}$ of an $n \times n$ $\{0,1\}$ matrix $A$ is now well understood as a graph $C^{*}$-algebra $C^{*}(E)$ of a finite directed graph $E$ with the vertex matrix $A\left(\mathcal{O}_{A} \cong \mathcal{O}_{B}\right.$ for the edge matrix $B$ of $\left.E\right)$. If $A$ has no zero rows or columns, the map $\phi_{A}: \mathcal{O}_{A} \rightarrow \mathcal{O}_{A}$ defined by

$$
\phi_{A}(x)=\sum_{j=1}^{n} s_{j} x s_{j}^{*}, \quad x \in \mathcal{O}_{A}
$$

is unital and completely positive, where $s_{j}$ 's, $1 \leqslant j \leqslant n$, are the partial isometries that generate $\mathcal{O}_{A}$. If $A$ is the edge matrix of $E, \phi_{A}$ corresponds to the unital completely positive map $\phi_{E}: C^{*}(E) \rightarrow C^{*}(E)$ given by

$$
\phi_{E}(x)=\sum_{e \in E^{1}} s_{e} x s_{e}^{*} .
$$

Received 13th January, 2004

The first author was partially supported by KOSEF R01-2000-000-00001-0. The second author was partially supported by KOSEF R01-2001-000-00001-0.

Copyright Clearance Centre, Inc. Serial-fee code: 0004-9727/04 \$A2.00+0.00. 
Then one can think of Voiculescu's topological entropy of $\phi_{E}$ (or $\phi_{A}$ ), and it turns out that if $E$ is a finite directed graph with no sinks

$$
\operatorname{ht}\left(\phi_{E}\right)=\log r\left(A_{E}\right),
$$

where $r(A)$ is the spectral radius of the edge matrix $A_{E}$ of $E$ (see $[15,4,7,3,5,14]$ ). One purpose of the present paper is to extend this result to a finite graph possibly with sinks, and the other is to provide a lower bound for $\operatorname{ht}\left(\phi_{E}\right)$ when $E$ is a locally finite irreducible infinite graph.

In Section 2, we review several definitions and properties of graph $C^{*}$-algebras, entropies, and Voiculescu's topological entropy of a completely positive map. Then Section 3 is devoted to obtaining ht $\left(\phi_{E}\right)$ for an arbitrary finite graph $E$ with the sinks $\mathcal{S}(E)$. To this end we consider another completely positive map $\psi_{E}$ on $C^{*}(E)$,

$$
\psi_{E}(x)=\phi_{E}(x)+\sum_{v \in \mathcal{S}(E)} p_{v} x p_{v}
$$

and show that

$$
\operatorname{ht}\left(\phi_{E}\right)=\mathrm{ht}\left(\psi_{E}\right)=\log r\left(A_{E}\right) .
$$

We first prove that $\log r\left(A_{E}\right) \leqslant \mathrm{ht}\left(\psi_{E}\right)$ by considering the topological entropy $h_{\text {top }}\left(X_{E_{S}}, \sigma\right)$ of the (compact) edge shift space $\left(X_{E_{\mathcal{S}}}, \sigma\right)$ of the finite graph $E_{\mathcal{S}}$ which we obtain from $E$ by adding a loop edge to each sink of $E$. For the reverse inequality ht $\left(\psi_{E}\right) \leqslant \log r\left(A_{E}\right)$ we shall modify the proof of [3, Theorem 1] to cover our general situation. Then $\mathrm{ht}\left(\phi_{E}\right)=\mathrm{ht}\left(\psi_{E}\right)$ is proved.

In Section 4 we consider a locally finite (irreducible) infinite graph $E$, and prove that the map $\phi_{E}$ given by

$$
\phi_{E}(x)=\sum_{e \in E^{1}} s_{e} x s_{e}^{*}, x \in C^{*}(E)
$$

is a (well defined) completely positive contraction. But ị this case the edge shift space $X_{E}$ may not be compact, so we shall consider Gureyic's compactification $\bar{X}_{E}$ of $X_{E}$ in order to find its topological entropy $h_{\text {top }}\left(\bar{X}_{E}\right)$ as a lower bound for $\operatorname{ht}\left(\phi_{E}\right)$. Note from [8] that $h_{\text {top }}\left(\bar{X}_{E}\right)=\sup _{E^{\prime}} h_{\text {top }}\left(X_{E^{\prime}}\right)$, where the supremum is taken over all the finite subgraphs of $\dot{E}$. Then it follows that $\mathrm{ht}\left(\phi_{E}\right)=\infty$ for many infinite irreducible graphs $E$. Nevertheless it would be interesting and important to know the exact value of $h t\left(\phi_{E}\right)$ when $\operatorname{ht}\left(\phi_{E}\right)$ is finite.

\section{Preliminaries}

2.1. GRAPHS AND GRAPH $C^{*}$-ALgEBRas. Let $E=\left(E^{0}, E^{1}, r, s\right)$ be a directed graph (or simply a graph) with a countable vertex set $E^{0}$ and a countable edge set $E^{1}$, where $r, s: E^{1} \rightarrow E^{0}$ are the range and source maps. If each vertex of $E$ emits and receives 
only finitely many edges, $E$ is called locally finite. By $\mathcal{S}(E)$ we denote the set of all sinks (vertices which emit no edges) of $E$. A sequence $\alpha=\left(\alpha_{1}, \alpha_{2}, \ldots, \alpha_{n}\right)$ of edges satisfying $r\left(\alpha_{i}\right)=s\left(\alpha_{i+1}\right), i=1, \ldots, n-1$, is called a (finite) path of length $|\alpha|=n$. We simply write $\alpha$ as $\alpha=\alpha_{1} \alpha_{2} \cdots \alpha_{n}$ and extend the maps $r, s$ to finite paths by $s(\alpha)$ $=s\left(\alpha_{1}\right), r(\alpha)=r\left(\alpha_{n}\right) . E^{n}$ will denote the set of all finite paths of length $n$ (each vertex is regarded as a finite path of length zero), and $E^{*}=\bigcup_{n=0}^{\infty} E^{n}$ denotes the set of all finite paths. Similarly an infinite path is defined to be an infinite sequence $\alpha=\alpha_{1} \alpha_{2} \cdots$ of edges with $r\left(\alpha_{i}\right)=s\left(\alpha_{i+1}\right), i=1,2, \ldots$. If a path $\alpha(|\alpha|>0)$ satisfies $s(\alpha)=r(\alpha)$ we call $\alpha$ a loop. A loop $\alpha$ is called a loop edge if $|\alpha|=1$.

For a graph $E$, a family $\left\{s_{e}, p_{v} \mid e \in E^{1}, v \in E^{0}\right\}$ of partial isometries $s_{e}$ (with mutually orthogonal ranges) and mutually orthogonal projections $p_{v}$ is called a CuntzKrieger E-family if it satisfies the following.

$$
\begin{aligned}
s_{e}^{*} s_{e} & =p_{r(e)}, \\
s_{e} s_{e}^{*} & \leqslant p_{s(e)}, \text { and } \\
p_{v} & =\sum_{s(e)=v} s_{e} s_{e}^{*} \text { if } 0<\left|s^{-1}(v)\right|<\infty .
\end{aligned}
$$

It is known (see $[2,12]$ for example) that there exists a universal $C^{*}$-algebra $C^{*}(E)$ (or $\left.C^{*}\left(s_{e}, p_{v}\right)\right)$ generated by a Cuntz-Krieger $E$-family $\left\{s_{e}, p_{v}\right\}$. We call $C^{*}(E)$ the graph $C^{*}$-algebra associated with $E$. It is useful to note that $\operatorname{span}\left\{s_{\alpha} s_{\beta}^{*} \mid \alpha, \beta \in E^{*}\right\}$ is dense in $C^{*}(E)$, where $s_{\alpha}=s_{\alpha_{1}} \cdots s_{\alpha_{k}}$ if $\alpha=\alpha_{1} \cdots \alpha_{k} \in E^{k}, k \geqslant 1$, and $s_{\alpha}=p_{v}$ if $\alpha=v \in E^{0}$.

2.2. Shift SPACE AND ENTropies. Let $\mathcal{A}$ be a finite set. Then a subset $X \subset \mathcal{A}^{\mathbb{N}}$ is called a (one-sided) shift space if there is a collection $\mathcal{F}$ of words over $\mathcal{A}$ such that $X$ is the set of all sequences $x$ in which no word of $\mathcal{F}$ can appear. By $\sigma_{X}$ we denote the shift map on $X$. Since $\mathcal{A}$ is finite (so compact in discrete topology), a shift space $X \subset \mathcal{A}^{\mathrm{N}}$ is a compact space and $\sigma_{X}$ is continuous, hence $\left(X, \sigma_{X}\right)$ carries the entropies which we review below.

(i) ([13, Definition 4.1.1] or $[10, \mathrm{p} .23])$ The entropy $h(X)$ of $X$ is defined by

$$
h(X)=\lim _{n \rightarrow \infty} \frac{1}{n} \log \left|W_{n}(X)\right|,
$$

where $W_{n}(X)$ is the set of all words of length $n$ that appear in a sequence of $X$. If $X \neq \emptyset$ we have $0 \leqslant h(X)<\log |\mathcal{A}|<\infty$ since $1 \leqslant\left|W_{n}(X)\right| \leqslant|\mathcal{A}|^{n}$. In particular, the full shift space $X_{n}=\mathcal{A}^{\mathrm{N}}(|\mathcal{A}|=n)$ has $h\left(X_{n}\right)=\log n$. If $X=\emptyset$ then $h(X)=-\infty$ by definition.

(ii) ([16, Chapter 7]) Let $T: X \rightarrow X$ be a continuous map on a compact space $X$. If $\mathcal{U}$ is an open cover of $X$ then so is $T^{-1} \mathcal{U}$. By $N(\mathcal{U})$ we denote the number of sets in a finite subcover of $\mathcal{U}$ with smallest cardinality. Then the entropy of $T$ relative to $\mathcal{U}$ is given by

$$
h_{\mathrm{top}}(T, \mathcal{U}):=\lim _{n \rightarrow \infty} \frac{1}{n} \log \left(N\left(\bigvee_{i=0}^{n-1} T^{-i} \mathcal{U}\right)\right)
$$


where $\mathcal{U} \vee \mathcal{V}$ denotes the join of $\mathcal{U}$ and $\mathcal{V}$, and the topological entropy of $(X, T)$ is defined to be

$$
h_{\text {top }}(X, T)=\sup _{\mathcal{U}} h_{\text {top }}(T, \mathcal{U})
$$

where the supremum is taken over all the open covers (or equivalently, over all the finite open covers) of $X$.

REMARK 2.1. (a) If $E$ is a finite graph we have the edge shift space

$$
X_{E}=\left\{\alpha=\left(\alpha_{i}\right) \in\left(E^{1}\right)^{\mathbf{N}} \mid r\left(\alpha_{i}\right)=s\left(\alpha_{i+1}\right), i \in \mathbb{N}\right\}
$$

(or the infinite path space) and the shift map $\sigma_{E}$ given by $\sigma_{E}(\alpha)_{i}=\alpha_{i+1}$ for each $i \in \mathbb{N}$. For $E$ with no infinite paths, we have $h\left(X_{E}\right)=-\infty$. Otherwise it is known [16, Theorem 7.13] that

$$
h_{\mathrm{top}}\left(X_{E}, \sigma_{E}\right)=h\left(X_{E}\right) \text {. }
$$

(b) Let $\Sigma_{E}\left(\subset\left(E^{1}\right)^{\mathbf{Z}}\right)$ be the two-sided shift space associated with a finite graph $E$. Then we know from $([10, \mathrm{p} .23])$ that

$$
h\left(X_{E}\right)=h\left(\Sigma_{E}\right) .
$$

We call a graph $E$ irreducible if for any two vertices $v, w$ there exists a finite path $\alpha$ with $s(\alpha)=v, r(\alpha)=w$. So a finite graph $E$ is irreducible if and only if its vertex matrix $V_{E}$ (or edge matrix $A_{E}$ ) is irreducible. Here a real, nonnegative square matrix $A=\left(A_{i j}\right)_{1 \leqslant i, j \leqslant n}$ is irreducible if for each $i, j$ there exists an $m \geqslant 1$ such that $\left(A^{m}\right)_{i j}>0$.

If $E$ is a finite graph, the vertex matrix $V_{E}$ has irreducible components $V_{1}, \ldots, V_{k}$ in the sense that each $V_{i}$ is an irreducible nonnegative square integer matrix and there exists a permutation matrix $P$ such that $P V_{E} P^{-1}$ is in a block triangular form with blocks $V_{1}, \ldots, V_{k}$ on its diagonal. Let $\lambda_{V_{i}}$ be the Perron-Frobenius eigenvalue of $V_{i}$. Then the Perron value $\lambda_{E}=\max _{1 \leqslant i \leqslant k} \lambda_{V_{i}}$ is the largest eigenvalue of $V_{E}$, hence $\lambda_{E}=r\left(V_{E}\right)$, the spectral radius of $V_{E}$ (see [13, Section 4.4]). One can write $E^{0}$ as the disjoint union of vertices $E_{i}^{0}(1 \leqslant i \leqslant k)$ so that each $V_{i}$ is a matrix with the index $E_{i}^{0}$. Let $E_{i}$ be the subgraph of $E$ with the vertex set $E_{i}^{0}$ and edge set $E_{i}^{1}=\left\{e \in E^{1} \mid s(e), r(e) \in E_{i}^{0}\right\}$, then $E_{i}$ is irreducible, and $E_{i}$ 's are called the irreducible components of $E$. If $E_{i}^{0}$ is a singleton and $\left|E_{i}^{1}\right|=1$, then $\log \lambda_{V_{i}}=0$, thus the subgraph $E_{i}$ makes no contribution to the value of $h\left(X_{E}\right)$ because

$$
h\left(X_{E}\right)=\log \lambda_{E}=\max _{1 \leqslant i \leqslant k} \log \lambda_{V_{i}}
$$

([13, Theorem 4.4.4]). On the other hand, it is easy to see that $r\left(A_{E}\right)=r\left(V_{E}\right)$. In fact, the rectangular matrices $R=\left(R_{e v}\right)_{e \in E^{1}, v \in E^{0}}, \quad S=\left(S_{v e}\right)_{v \in E^{0}, e \in E^{1}}$, where

$$
R_{e v}=\left\{\begin{array}{ll}
1, & \text { if } r(e)=v, \\
0, & \text { otherwise }
\end{array} \quad \text { and } \quad S_{v e}= \begin{cases}1, & \text { if } s(e)=v, \\
0, & \text { otherwise }\end{cases}\right.
$$

satisfy $R S=A_{E}$ and $S R=V_{E}$, which implies that $\lambda$ is an eigenvalue of $V_{E}$ if and only if $\lambda$ is an eigenvalue of $A_{E}$. Hence we have the following. 
Proposition 2.2. Let $E$ be a finite graph and $X_{E}$ be the one-sided shift space associated with $E$. Then

$$
h\left(X_{E}\right)=\log \lambda_{E}=\log r\left(A_{E}\right)
$$

where $\lambda_{E}$ is the Perron value of the edge matrix $A_{E}$ (or the vertex matrix $V_{E}$ ) of $E$ and $r\left(A_{E}\right)$ is the spectral radius of $A_{E}$.

2.3. TOPOlOgiCAL ENTROPY OF A COMPLETELY POSITIVE MAP. We briefly review the definition of topological entropy for a completely positive map of a $C^{*}$-algebra which was first defined for automorphisms of unital nuclear $C^{*}$-algebras by Voiculescu [15] and then extended to automorphisms of exact $C^{*}$-algebras by Brown [4]. See also [7] and [3] for the following definition of topological entropy for a completely positive map.

Let $\pi: A \rightarrow B(H)$ be a faithful representation of a $C^{*}$-algebra $A$ and $P f(A)$ be the set of all finite subsets of $A$. For $\omega \in P f(A)$ and $\delta>0$, we put

$$
\begin{aligned}
& \operatorname{CPA}(\pi, A):=\{(\phi, \psi, B) \mid \phi: A \rightarrow B, \psi: B \rightarrow B(H) \rightarrow \\
&\text { contractive completely positive maps, } \operatorname{dim} B<\infty\}, \\
& \operatorname{rcp}(\pi, \omega, \delta):= \inf \{\operatorname{rank}(B) \mid(\phi, \psi, B) \in \operatorname{CPA}(\pi, A),\|\psi \circ \phi(x)-\pi(x)\|<\delta, \\
&\text { for all } x \in \omega\},
\end{aligned}
$$

where $\operatorname{rank}(B):=$ the dimension of a maximal Abelian subalgebra of $B$.

It is well known [9] that every exact $C^{*}$-algebra $A$ is nuclearly embeddable, that is, there exists a faithful representation $\pi: A \rightarrow B(H)$ such that for each finite subset $\omega \subset A$ and $\delta>0$ there is $(\phi, \psi, B) \in \operatorname{CPA}(\pi, A)$ with $\psi \circ \phi$ close to $\pi$ within $\delta$ on $\omega$. Moreover the value $\operatorname{rcp}(\pi, \omega, \delta)$ is independent of the choice of $\pi$ (see $[4,3]$ ). Since graph $C^{*}$-algebras $C^{*}(E)$ are nuclear (see $[11$, p. 193]) we may write $\operatorname{rcp}(\omega, \delta)$ for $\operatorname{rcp}(\pi, \omega, \delta)$ assuming $C^{*}(E) \subset B(H)$ for a Hilbert space $H$.

Definition 2.3: ([4, 3]) Let $A \subset B(H)$ be a $C^{*}$-algebra and $\Phi: A \rightarrow A$ be a completely positive map. Then we define

$$
\begin{aligned}
\operatorname{ht}(\Phi, \omega, \delta) & =\limsup _{n \rightarrow \infty} \frac{1}{n} \log \left(\operatorname{rcp}\left(\omega \cup \Phi(\omega) \cup \cdots \cup \Phi^{n-1}(\omega), \delta\right)\right), \\
\operatorname{ht}(\Phi, \omega) & =\sup _{\delta>0} \operatorname{ht}(\Phi, \omega, \delta) \\
\operatorname{ht}(\Phi) & =\sup _{\omega \in P f(A)} \operatorname{ht}(\Phi, \omega) .
\end{aligned}
$$

ht $(\Phi)$ is called the topological entropy of $\Phi$.

REMARK 2.4. We refer the reader to $[3,4]$, and [7] for the following useful properties. Let $\Phi: A \rightarrow A$ be a completely positive map on an exact $C^{*}$-algebra $A$. 
(a) If $\theta: A \rightarrow B$ is a $C^{*}$-isomorphism then

$$
\operatorname{ht}(\Phi)=\operatorname{ht}\left(\theta \Phi \theta^{-1}\right) .
$$

(b) Let $\tilde{A}$ be the unital $C^{*}$-algebra obtained by adjoining a unit. Let $\widetilde{\Phi}: \widetilde{A} \rightarrow \widetilde{A}$ be the extension of $\Phi$. Then

$$
\operatorname{ht}(\widetilde{\Phi})=\operatorname{ht}(\Phi) .
$$

(c) If $A_{0}$ is a $\Phi$-invariant $C^{*}$-subalgebra of $A$, then

$$
\operatorname{ht}\left(\left.\Phi\right|_{A_{0}}\right) \leqslant \operatorname{ht}(\Phi) .
$$

(d) If $\left\{\omega_{k}\right\}$ is an increasing sequence of finite subsets in $A$ such that the linear span of the set $\bigcup_{k, l \in \mathbf{Z}^{+}} \Phi^{l}\left(\omega_{k}\right)$ is dense in $A$, then

$$
h t(\Phi)=\operatorname{supht}_{k}\left(\Phi, \omega_{k}\right)
$$

(e) Let $T: X \rightarrow X$ be a continuous map on a compact metric space $X$. Then $\operatorname{ht}\left(T^{*}\right)=h_{\text {top }}(X, T)$, where $T^{*}: C(X) \rightarrow C(X)$ is the completely positive map given by $T^{*}(f)=f \circ T, f \in C(X)$.

\section{FINITE GRAPHS}

In this section we consider the following two completely positive maps $\phi_{E}, \psi_{E}$ on the graph $C^{*}$-algebra $C^{*}(E)$ associated with a finite graph $E$,

$$
\begin{aligned}
& \phi_{E}(x)=\sum_{e \in E^{1}} s_{e} x s_{e}^{*} \\
& \psi_{E}(x)=\sum_{e \in E^{1}} s_{e} x s_{e}^{*}+\sum_{v \in \mathcal{S}(E)} p_{v} x p_{v} .
\end{aligned}
$$

We call $\phi_{E}$ the canonical completely positive map of $C^{*}(E)$ which is not unital if $E$ contains a sink while $\psi_{E}$ is always. A computation shows that

$$
\psi_{E}^{n}(x)=\sum_{|\mu|=n} s_{\mu} x s_{\mu}^{*}+\sum_{\substack{0<|\eta|<n \\ r(\eta) \in \mathcal{S}(E)}} s_{\eta} x s_{\eta}^{*}+\sum_{v \in \mathcal{S}(E)} p_{v} x p_{v} .
$$

Hence if $E$ has no infinite paths then there exists an $N$ such that the first term $\sum_{|\mu|=n} s_{\mu} x s_{\mu}{ }^{*}$ vanishes and $\psi_{E}^{n}(x)=\psi_{E}^{N}(x)$ whenever $n>N$. Thus it follows that ht $\left(\psi_{E}\right)=0$. But the edge matrix $A_{E}$ has no nonzero irreducible components and so its Perron value is 0 . Hence we see from Proposition 2.2 that $\log r\left(A_{E}\right)=-\infty$.

We now compute ht $\left(\psi_{E}\right)$ (and ht $\left(\phi_{E}\right)$ ) for $E$ which contains an infinite path. 
THEOREM 3.1. Let $E$ be a finite graph with the edge matrix $A_{E}$. If $E$ contains an infinite path then

$$
\operatorname{ht}\left(\psi_{E}\right)=\log r\left(A_{E}\right),
$$

where $r\left(A_{E}\right)$ is the spectral radius of $A_{E}$.

Let $\mathcal{D}_{E}$ be the commutative $C^{*}$-subalgebra of $C^{*}(E)$ generated by projections of the form $p_{\mu}=s_{\mu} s_{\mu}^{*}, \mu \in E^{*}$. Then $\mathcal{D}_{E}$ is $\psi_{E}$-invariant and

$$
\mathcal{D}_{E}=\overline{\operatorname{span}}\left\{p_{\mu}=s_{\mu} s_{\mu}^{*} \in C^{*}(E) \mid \mu \in E^{*}\right\} .
$$

Now we seek a shift space $\left(X, \sigma_{X}\right)$ such that there exists an isomorphism $w: \mathcal{D}_{E} \rightarrow C(X)$ satisfying $w\left(\psi_{E} \mid \mathcal{D}_{E}\right) w^{-1}=\sigma_{X}^{*}$ from which we deduce that $h(X) \leqslant \mathrm{ht}\left(\psi_{E}\right)$. Let $E_{\mathcal{S}}$ be the graph obtained from $E$ by adding a loop edge $e_{v}$ to each $\operatorname{sink} v \in \mathcal{S}(E)$, that is,

$$
E_{\mathcal{S}}^{0}=E^{0}, \quad E_{\mathcal{S}}^{1}=E^{1} \cup\left\{e_{v} \mid s\left(e_{v}\right)=r\left(e_{v}\right)=v, v \in \mathcal{S}(E)\right\}
$$

and consider the shift space $X_{E_{S}}$ of infinite paths. Then the cylinder sets $[\mu]=\{\mu \alpha \mid$ $\left.\mu \alpha \in X_{E_{S}}\right\}, \mu \in E_{\mathcal{S}}^{*}$, are both open and compact, and form a basis for the subspace topology of the compact space $X_{E_{S}} \subset\left(E_{\mathcal{S}}^{1}\right)^{\mathrm{N}}$. Hence the characteristic functions $\chi_{[\mu]}$, $\mu \in E_{\mathcal{S}}^{*}$, are continuous on $X_{E_{S}}$. Moreover applying the Stone-Weierstrass theorem one sees that the linear span of the characteristic functions $\left\{\chi_{[\mu]} \mid \mu \in E_{\mathcal{S}}^{n}, n \in \mathbb{N}\right\}$ is dense in $C\left(X_{E_{S}}\right)$. Then as in [6, Proposition 2.5] and [14, Corollary 7.2], one obtains the following.

Lemma 3.2. The linear map $w: \mathcal{D}_{E} \rightarrow C\left(X_{E_{S}}\right)$ given by

$$
w\left(p_{\mu}\right)= \begin{cases}\chi_{[\mu]}, & \text { if }|\mu| \geqslant 1, \\ \chi_{\left[e_{v}\right]}, & \text { if } \mu=v \in \mathcal{S}(E)\end{cases}
$$

is a $*$-isomorphism such that $w\left(\left.\psi_{E}\right|_{\mathcal{D}(E)}\right) w^{-1}=\left(\sigma_{X_{E_{S}}}\right)^{*}$.

Proposition 3.3. $h_{\text {top }}\left(X_{E_{S}}, \sigma_{X_{E_{S}}}\right)=\operatorname{ht}\left(\left.\psi_{E}\right|_{\mathcal{D}_{E}}\right) \leqslant \mathrm{ht}\left(\psi_{E}\right)$.

Proof: By Remark 2.4(e), we have $h_{\text {top }}\left(X_{E_{S}}, \sigma_{X_{E_{S}}}\right)=\operatorname{ht}\left(\left(\sigma_{X_{E_{S}}}\right)^{*}\right)$. Also Remark 2.4(a) and Lemma 3.2 imply that ht $\left(\left(\sigma_{X_{E_{S}}}\right)^{*}\right)=\operatorname{ht}\left(\left.\psi_{E}\right|_{\mathcal{D}_{E}}\right)$. The last inequality follows from Remark 2.4(c).

\section{PROPOSITION 3.4 .}

(a) $h\left(X_{E}\right)=h\left(X_{E_{S}}\right)$.

(b) Let $G$ be the graph obtained from $E$ by removing vertices $v$ with $s^{-1}(v)$ consisting of a loop edge and all edges in $r^{-1}(v)$ and then adding a loop edge to each newly formed sink, if any. Then $h\left(X_{E}\right)=h\left(X_{G}\right)$.

Proof: (a) immediately follows from Proposition 2.2 and the arguments before it. For (b), apply (a) and the arguments before Proposition 2.2 repeatedly. 
Proposition 3.5. $\log r\left(A_{E}\right)=h\left(X_{E}\right) \leqslant \mathrm{ht}\left(\psi_{E}\right)$.

Proof: $h\left(X_{E}\right)=h\left(X_{E_{\mathcal{S}}}\right)$ by Proposition 3.4(a), and $h\left(X_{E_{\mathcal{S}}}\right)=h_{\text {top }}\left(X_{E_{\mathcal{S}}}, \sigma_{X_{E_{\mathcal{S}}}}\right)$ by Remark 2.1.(a). Then Proposition 3.3 proves the assertion.

For the proof of the reverse inequality ht $\left(\psi_{E}\right) \leqslant \log r\left(A_{E}\right)$, we modify the proof in [3] according to our general situation. But we have to deal with more complicated situation due to the existence of sinks which do not appear in case of [3], so we present a proof here. Put

$$
W(n):=E^{n} \cup\left\{\mu \in \bigcup_{k=0}^{n-1} E^{k} \mid r(\mu) \in \mathcal{S}(E)\right\} .
$$

Then there is a one to one correspondence between $W(n)$ and the set $\left(E_{\mathcal{S}}\right)^{n}$ of finite paths of length $n$ in $E_{\mathcal{S}}$, and so the following lemma is an immediate consequence of Proposition 3.4(a).

LEMMA 3.6. $\lim _{n \rightarrow \infty}(1 / n) \log |W(n)|=\log r\left(A_{E}\right)$.

As in [3] we define a map $\rho_{m}: C^{*}(E) \rightarrow M_{|W(m)|} \otimes C^{*}(E)$ by

$$
\rho_{m}(x):=\sum_{\mu, \nu \in W(m)} e_{\mu \nu} \otimes s_{\mu}^{*} x s_{\nu} .
$$

LEMMA 3.7. $\rho_{m}$ is an injective *-homomorphism.

Proof: Since $\sum_{\mu \in W(m)} s_{\mu} s_{\mu}^{*}=I$, the unit of $C^{*}(E)$, it easily follows that $\rho_{m}$ is a *-homomorphism. To see that $\rho_{m}$ is injective, suppose $\rho_{m}(x)=0$ (in [3], $C^{*}(E)$ was simple). Then $s_{\mu}^{*} x s_{\nu}=0$ for all $\mu, \nu \in W(m)$. Thus for each pair of vertices $v, w \in E^{0}$,

$$
\sum_{\substack{\mu \in W(m), s(\mu)=v \\ \nu \in W(m), s(\nu)=w}} s_{\mu} s_{\mu}^{*} x s_{\nu} s_{\nu}^{*}=0,
$$

which implies that $p_{v} x p_{w}=0$ since

$$
p_{v}=\sum_{\mu \in W(m), s(\mu)=v} s_{\mu} s_{\mu}^{*}
$$

Therefore $x=0$ and $\rho_{m}$ is injective.

LeMmA 3.8. Let $n \in \mathbb{N},|\beta| \leqslant|\alpha| \leqslant n_{0}$, and $m \geqslant n+n_{0}$. Then for each $0 \leqslant l \leqslant n-1$,

$$
\rho_{m}\left(\psi_{E}^{l}\left(s_{\alpha} s_{\beta}^{*}\right)\right)=\sum_{\mu \in W(|\alpha|-|\beta|)} X(\mu, \alpha, \beta, l, m) \otimes s_{\mu}
$$

for some partial isometries $X(\mu, \alpha, \beta, l, m)$. 
Proof: Note first that if $\mu, \nu \in W(m)$ and $|\mu| \neq|\nu|$ then $s_{\mu}^{*} s_{\nu}=0$. Also if $\mu, \nu \in E^{*}, r(\mu) \in \mathcal{S}(E)$, and $|\mu|<|\nu|$ then $s_{\mu}^{*} s_{\nu}=0$. Then from the formula (1)

$$
\begin{aligned}
\rho_{m}\left(\psi_{E}^{l}\left(s_{\alpha} s_{\beta}^{*}\right)\right) & =\sum_{\eta \in W(l)} \rho_{m}\left(s_{\eta} s_{\alpha} s_{\beta}^{*} s_{\eta}^{*}\right) \\
& =\sum_{\eta \in W(l)} \sum_{\mu, \nu \in W(m)} e_{\mu \nu} \otimes s_{\mu}^{*} s_{\eta} s_{\alpha} s_{\beta}^{*} s_{\eta}^{*} s_{\nu} \\
& =\sum_{\mu \in W(|\alpha|-|\beta|)} \sum_{\substack{\eta \alpha \mu^{\prime}, \eta \beta \mu^{\prime} \mu \in W(m) \\
\eta \in W(l)}} e_{\eta \alpha \mu^{\prime}, \eta \beta \mu^{\prime} \mu} \otimes s_{\mu},
\end{aligned}
$$

and $X(\mu, \alpha, \beta, l, m):=\sum_{\substack{\eta \alpha \mu^{\prime}, \eta \beta \mu^{\prime} \mu \in W(m) \\ \eta \in W(l)}} e_{\eta \alpha \mu^{\prime}, \eta \beta \mu^{\prime} \mu}$ is a partial isometry with the range projection $X(\mu, \alpha, \beta, l, m) X(\mu, \alpha, \beta, l, m)^{*}=\sum_{\substack{\eta \alpha \mu^{\prime} \in W(m) \\ \eta \in W(l)}} e_{\eta \alpha \mu^{\prime}, \eta \alpha \mu^{\prime}}$.

For each $n_{0} \geqslant 1$, put

$$
\omega\left(n_{0}\right):=\left\{s_{\alpha} s_{\beta}^{*}|| \beta|\leqslant| \alpha \mid \leqslant n_{0}\right\} .
$$

Then the following proposition implies that

$$
\operatorname{ht}\left(\psi_{E}\right) \leqslant \log r\left(A_{E}\right),
$$

since the linear span of the set $\bigcup_{k \geqslant 1}\left(\omega(k) \cup \omega(k)^{*}\right)$ is dense in $C^{*}(E)$ (Remark 2.4.(d)).

Proposition 3.9. Let $n_{0} \geqslant 1$ and $\delta>0$. Then

$$
\operatorname{ht}\left(\psi_{E}, \omega\left(n_{0}\right), \delta\right)=\underset{n}{\limsup } \frac{1}{n} \log \operatorname{rcp}\left(\bigcup_{i=0}^{n-1} \psi_{E}^{i}\left(\omega\left(n_{0}\right)\right), \delta\right) \leqslant \log r\left(A_{E}\right) .
$$

Proof: Let $H$ be a Hilbert space on which $C^{*}(E)$ acts faithfully. Since $C^{*}(E)$ is nuclear, there exists $\left(\phi_{0}, \psi_{0}, M_{m_{0}}\right) \in \operatorname{CPA}\left(i d_{C^{*}(E)}, C^{*}(E)\right)$ such that

$$
\left\|\psi_{0} \phi_{0}\left(s_{\gamma}\right)-s_{\gamma}\right\|<\frac{\delta}{\left|W\left(n_{0}\right)\right|}, \quad \gamma \in W\left(n_{0}\right) .
$$

Now for $n \geqslant 1$, let $m=m(n)=n+n_{0}$ and $B=M_{|W(m)|} \otimes M_{m_{0}}$. Then by Arveson's extension theorem (see $\left[4\right.$, p. 349]) the $*$-isomorphism $\rho_{m}^{-1}: \rho_{m}\left(C^{*}(E)\right) \rightarrow C^{*}(E)$ extends to a unital completely positive map

$$
\Psi_{m}: M_{|W(m)|} \otimes C^{*}(E) \rightarrow B(H) .
$$

Now consider the completely positive maps $\phi$ and $\psi$ given by

$$
\phi=\left(i d \otimes \phi_{0}\right) \rho_{m}: C^{*}(E) \rightarrow B \quad \text { and } \quad \psi=\Psi_{m}\left(i d \otimes \psi_{0}\right): B \rightarrow B(H) .
$$




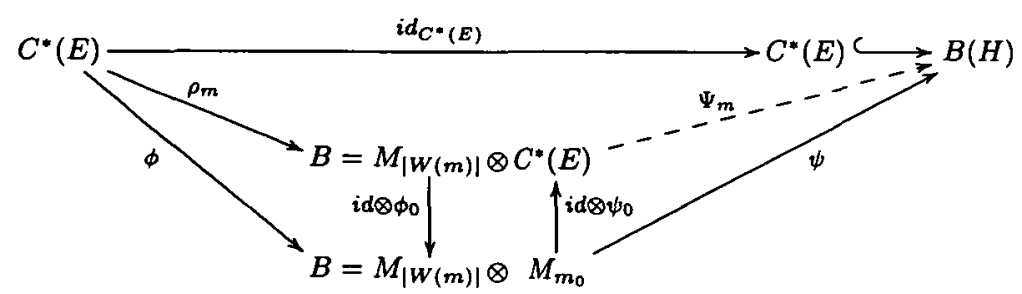

Let $a=s_{\alpha} s_{\beta}^{*} \in \omega\left(n_{0}\right)$. Then by Lemma 3.8 there exist partial isometries $X(\mu)$ $=X(\mu, \alpha, \beta, l, m)$ such that

$$
\rho_{m} \psi_{E}^{l}(a)=\sum_{\mu \in W(|\alpha|-|\beta|)} X(\mu) \otimes s_{\mu}
$$

Then as in [3] it follows from (2) and (3) that

$$
\left\|\psi \phi\left(\psi_{E}^{l}(a)\right)-\psi_{E}^{l}(a)\right\|<\left|W\left(n_{0}\right)\right| \cdot \frac{\delta}{\left|W\left(n_{0}\right)\right|}=\delta
$$

Therefore

$$
\operatorname{rcp}\left(\bigcup_{i=0}^{n-1} \psi_{E}^{i}\left(\omega\left(n_{0}\right)\right), \delta\right) \leqslant m_{0}|W(m)|=m_{0}\left|W\left(n+n_{0}\right)\right|
$$

and so $\limsup _{n}(1 / n) \log \operatorname{rcp}\left(\bigcup_{i=0}^{n-1} \psi_{E}^{i}\left(\omega\left(n_{0}\right)\right), \delta\right) \leqslant \log r\left(A_{E}\right)$ (by Lemma 3.6).

COROLLARY 3.10. Let $E$ be a finite directed graph and $G$ be a subgraph of $E$ obtained by removing sinks and edges going into them. Then

$$
\operatorname{ht}\left(\psi_{E}\right)=\operatorname{ht}\left(\psi_{G}\right)
$$

In the rest of the section we show that $\operatorname{ht}\left(\phi_{E}\right)=\operatorname{ht}\left(\psi_{E}\right)$.

LEMMA 3.11. $\psi_{E}^{l}(x)=\phi_{E}^{l}(x)+\psi_{E}^{l-1}\left(\sum_{v \in S(E)} p_{v} x p_{v}\right), l \in \mathbb{N}$.

ProOF: Since $\psi_{E}(x)=\phi_{E}(x)+\sum_{v \in \mathcal{S}(E)} p_{v} x p_{v}$, we have

$$
\begin{aligned}
\psi_{E}^{2}(x) & =\phi_{E}\left(\phi_{E}(x)+\sum_{v \in \mathcal{S}(E)} p_{v} x p_{v}\right)+\sum_{w \in \mathcal{S}(E)} p_{w}\left(\phi_{E}(x)+\sum_{v \in \mathcal{S}(E)} p_{v} x p_{v}\right) p_{w} \\
& =\phi_{E}^{2}(x)+\phi_{E}\left(\sum_{v \in \mathcal{S}(E)} p_{v} x p_{v}\right)+\sum_{w \in \mathcal{S}(E)} p_{w}\left(\sum_{v \in \mathcal{S}(E)} p_{v} x p_{v}\right) p_{w} \\
& =\phi_{E}^{2}(x)+\psi_{E}\left(\sum_{v \in \mathcal{S}(E)} p_{v} x p_{v}\right)
\end{aligned}
$$

For $l \geqslant 3$, use induction on $l$. 
Let $\phi_{L}: C^{*}(E) \rightarrow C^{*}(E)$ be the completely positive map given by $\phi_{L}(x)$ $=\sum_{\substack{e \in E^{1} \\ r(e) \notin \mathcal{S}(E)}} s_{e} x s_{e}^{*}$.

PROPOSITION 3.12. $\operatorname{ht}\left(\phi_{L}\right)=\operatorname{ht}\left(\phi_{E}\right) \leqslant \operatorname{ht}\left(\psi_{E}\right)$.

Proof: Let $\delta>0, n \in \mathbb{N}$, and let $\omega \subset C^{*}(E)$ be a finite set of the elements of the form $s_{\alpha} s_{\beta}^{*}, \alpha, \beta \in E^{*}$ such that $\left\{p_{v} \mid v \in \mathcal{S}(E)\right\} \subset \omega$. Then choose an element $\left(\psi_{1}, \psi_{2}, B\right) \in \mathrm{CPA}\left(i d_{C^{*}(E)}, C^{*}(E)\right)$ satisfying $\operatorname{rank}(B)=\operatorname{rcp}\left(\bigcup_{j=0}^{n-1} \psi_{E}^{j}(\omega), \delta\right)$. If $x \in \omega$, $1 \leqslant l \leqslant n-1$, then by the above lemma

$$
\begin{aligned}
\left\|\psi_{2} \psi_{1}\left(\phi_{E}^{l}(x)\right)-\phi_{E}^{l}(x)\right\| & \leqslant\left\|\psi_{2} \psi_{1}\left(\psi_{E}^{l}(x)\right)-\psi_{E}^{l}(x)\right\| \\
& +\left\|\psi_{2} \psi_{1}\left(\psi_{E}^{l-1}\left(\sum_{v \in \mathcal{S}(E)} p_{v} x p_{v}\right)\right)-\psi_{E}^{l-1}\left(\sum_{v \in \mathcal{S}(E)} p_{v} x p_{v}\right)\right\| \leqslant 2 \delta
\end{aligned}
$$

and $\operatorname{rcp}\left(\bigcup_{j=0}^{n-1} \phi_{E}^{j}(\omega), 2 \delta\right) \leqslant \operatorname{rcp}\left(\bigcup_{j=0}^{n-1} \psi_{E}^{j}(\omega), \delta\right)$. Thus we have ht $\left(\phi_{E}\right) \leqslant \operatorname{ht}\left(\psi_{E}\right)$.

To prove the first equality, note that $\phi_{E}^{l}(x)=\phi_{L}^{l}(x)$ if $x=s_{\alpha} s_{\beta}{ }^{*} \in \omega$ with $|\alpha|+|\beta|$ $>0$, and $\phi_{L}^{l}(x)=0$ if $x=p_{v}, v \in \mathcal{S}(E)$. Thus

$$
\bigcup_{i=0}^{n-1} \phi_{L}^{i}(\omega) \subseteq \bigcup_{i=0}^{n-1} \phi_{E}^{i}(\omega) \cup\{0\},
$$

and hence $\operatorname{ht}\left(\phi_{L}\right) \leqslant \operatorname{ht}\left(\phi_{E}\right)$. Put $\bar{\omega}:=\omega \cup \phi_{E}(\omega)$. From definitions of $\phi_{E}$ and $\phi_{L}$ it is easily seen that $\phi_{E}^{l}(x)=\phi_{L}^{l-1}\left(\phi_{E}(x)\right), l \geqslant 1$. Thus

$$
\bigcup_{i=0}^{n-1} \phi_{E}^{i}(\omega) \subseteq \bigcup_{i=0}^{n-1} \phi_{L}^{i}(\bar{\omega})
$$

which also shows that $\operatorname{ht}\left(\phi_{E}\right) \leqslant \operatorname{ht}\left(\phi_{L}\right)$.

Note that the commutative $C^{*}$-subalgebra

$$
\mathcal{D}_{E}^{\prime}:=\overline{\operatorname{span}}\left\{p_{\mu}=s_{\mu} s_{\mu}{ }^{*} \mid \mu \in E^{*}, r(\mu) \notin \mathcal{S}(E)\right\} .
$$

of $C^{*}(E)$ is $\phi_{L}$-invariant and so ht $\left(\left.\phi_{L}\right|_{\mathcal{D}_{E}^{\prime}}\right) \leqslant \mathrm{ht}\left(\phi_{L}\right)$.

Proposition 3.13. ht $\left(\phi_{E}\right)=\operatorname{ht}\left(\psi_{E}\right)$.

Proof: Let $G$ be the graph obtained from $E$ by removing the sinks $S(E)$ and the edges going into them. Then as in Lemma 3.2, one can show that there is an isomorphism $w^{\prime}: \mathcal{D}_{E}^{\prime} \rightarrow C\left(X_{G}\right)$ such that

$$
\sigma_{G}^{*}=w^{\prime}\left(\left.\phi_{L}\right|_{\mathcal{D}_{E}^{\prime}}\right)\left(w^{\prime}\right)^{-1}
$$

where $\sigma_{G}$ is the shift map on $X_{G}$. Thus ht $\left(\left.\phi_{L}\right|_{\mathcal{D}_{E}^{\prime}}\right)=h\left(X_{G}\right)$. Consequently,

$$
\operatorname{ht}\left(\psi_{E}\right)=\log r\left(A_{E}\right)=h\left(X_{E}\right)=h\left(X_{G}\right)=\operatorname{ht}\left(\left.\phi_{L}\right|_{\mathcal{D}_{E}^{\prime}}\right) \leqslant \operatorname{ht}\left(\phi_{E}\right)
$$

by Theorem 3.1, Corollary 3.10, and Proposition 3.12. 
EXAMPLE 3.14. The Toeplitz algebra $\mathcal{T}$ can be viewed as the graph $C^{*}$-algebra $C^{*}(E)$ of $\left.E=\left(E^{0}=\{v, w\}, E^{1}=\{e, f)\right\}\right)$, where $s(e)=r(e)=s(f)=v, r(f)=w$. In fact, if $\left\{s_{e}, s_{f}, p_{v}, p_{w}\right\}$ is a Cuntz-Krieger $E$-family generating $C^{*}(E)$ the element $U:=s_{e}+s_{f}$ satisfies that $U^{*} U=I=p_{v}+p_{w}, U U^{*}=p_{v}, U^{*} U-U U^{*}=p_{w}, U^{2} U^{*}=s_{e}$, and $U-U^{2} U^{*}=s_{f}$. Thus $C^{*}(E)=C^{*}\{U\}$ and so by Coburn's theorem $\mathcal{T}=C^{*}\{U\}=C^{*}(E)$. Since $U^{*} U=I$, the linear span of the set $\left\{U^{m}\left(U^{*}\right)^{n} \mid m, n \geqslant 0\right\}$ is dense in $C^{*}(E)$, and one can show that $\phi_{E}(x)=U x U^{*}$ for each $x$ of the form $U^{m}\left(U^{*}\right)^{n}$. Thus $\phi_{E}$ is the endomorphism $\operatorname{Ad}(U)$ on $\mathcal{T}$. Since $r\left(A_{E}\right)=1$, it follows from Theorem 3.1 and Proposition 3.13 that ht $\left(\phi_{E}\right)=\log r\left(A_{E}\right)=0$. Thus ht $(\operatorname{Ad}(U))=0$.

\section{INFINITE GRAPHS}

In this section we consider the topological entropy of $\phi_{E}$ for an infinite graph $E$.

Proposition 4.1. Let $E$ be a locally finite infinite graph and let $C^{*}(E)$ $=C^{*}\left(s_{e}, p_{v}\right)$ be its associated $C^{*}$-algebra. Then the sum $\sum_{e \in E^{1}} s_{e} x s_{e}^{*}$ exists for each $x \in C^{*}(E)$ and the map $\phi_{E}: C^{*}(E) \rightarrow C^{*}(E)$ given by

$$
\phi_{E}(x)=\sum_{e \in E^{1}} s_{e} x s_{e}^{*}, x \in C^{*}(E)
$$

is a completely positive contraction.

Proof: For an $x \in C^{*}(E)$ and $\varepsilon>0$, choose a finite subgraph $F$ of $E$ and an element $z=\sum_{\alpha, \beta \in F^{*}} \lambda_{\alpha \beta} s_{\alpha} s_{\beta^{*}}\left(\lambda_{\alpha \beta} \in \mathbb{C}\right)$ such that $\|x-z\|<\varepsilon$. Put $E^{1}=\left\{e_{1}, e_{2}, \ldots\right\}$. Then by the local finiteness of $E$ there is a number $N$ such that

$$
F^{1} \cup\left\{e \in E^{1} \mid r(e) \in F^{0}\right\} \subset E_{N}^{1}:=\left\{e_{1}, e_{2}, \ldots, e_{N}\right\},
$$

so that $z p_{r\left(e_{k}\right)}=0$ for $k \geqslant N+1$. For any finite set $E^{\prime}$ of edges, let $V_{E^{\prime}}:=\{r(e) \mid e$ $\left.\in E^{\prime} \backslash E_{N}^{1}\right\}$ and $P:=\sum_{v \in V_{E^{\prime}}} p_{v}$. Then $\|x P\|=\|(x-z) P\|<\varepsilon$, and

$$
\left\|\sum_{e \in E^{\prime} \backslash E_{N}^{1}} s_{e} x s_{e}^{*}\right\|=\left\|\sum_{e \in E^{\prime} \backslash E_{N}^{1}} s_{e}(x P)^{*}(x P) s_{e}^{*}\right\|^{1 / 2} \leqslant\|x P\|<\varepsilon .
$$

Thus if $E^{\prime}, E^{\prime \prime}$ are two finite sets of edges with $E_{N}^{1} \subset E^{\prime} \cap E^{\prime \prime}$, then

$$
\left\|\sum_{e \in E^{\prime}} s_{e} x s_{e}^{*}-\sum_{e \in E^{\prime \prime}} s_{e} x s_{e}^{*}\right\| \leqslant\left\|\sum_{e \in E^{\prime} \backslash E_{N}^{1}} s_{e} x s_{e}^{*}\right\|+\left\|\sum_{e \in E^{\prime \prime} \backslash E_{N}^{1}} s_{e} x s_{e}^{*}\right\|<2 \varepsilon,
$$

which shows that the sum $\sum_{e \in E^{1}} s_{e} x s_{e}^{*}$ exists and the map $\phi_{E}$ is well defined. To see that $\phi_{E}$ is a contractive completely positive map, consider a sequence of completely positive 
maps $\phi_{n}: C^{*}(E) \rightarrow C^{*}(E)$ given by $\phi_{n}(x)=\sum_{i=1}^{n} s_{e_{i}} x s_{e_{i}}{ }^{*}$. If $x \geqslant 0$ then $\phi_{E}(x) \geqslant 0$ as the limit of positive elements $\phi_{n}(x)$ in norm. The same argument also proves that $\phi_{E}$ is completely positive. Since each $\phi_{n}$ is contractive we have $\left\|\phi_{E}\right\| \leqslant 1$.

The (one-sided) shift space $X_{E}$ may not be compact for an infinite graph $E$, which makes the definition $h_{\text {top }}\left(X_{E}\right)$ meaningless. This leads Gurevic [8] to consider a compactification of $X_{E}$ : Identify the edge set $E^{1}=\left\{e_{n}\right\}_{n \in \mathbb{N}}$ with the metric space $\{1,(1 / 2),(1 / 3), \ldots\} \subset[0,1]$ by $e_{n} \mapsto(1 / n)$, and let $\bar{E}^{1}:=E^{1} \cup\{0\}$ $=\{0,1,(1 / 2),(1 / 3), \ldots\}$ be the one-point compactification. Then $X_{E}$ becomes the subspace of the product space $\left(\bar{E}^{1}\right)^{\mathrm{N}}$ with the closure $\bar{X}_{E}$, where the metric

$$
d\left(\left(x_{n}\right),\left(y_{n}\right)\right)=\sum_{n=1}^{\infty} \frac{1}{2^{n}}\left|x_{n}-y_{n}\right|, \quad x_{n}, y_{n} \in \bar{E}^{1}
$$

is compatible with the product topology. The shift map $\bar{\sigma}_{E}:=\sigma_{\bar{X}_{E}}$ on the compact metric space $\bar{X}_{E}$ now has a well-defined topological entropy. Similarly we have the compact metric space $\bar{\Sigma}_{E} \subset\left(\bar{E}^{1}\right)^{\mathbf{z}}$ and the shift map $\bar{\sigma}_{E}:=\sigma_{\bar{\Sigma}_{E}}$. We use the same notation for two shift maps.

LEMmA 4.2. If $E$ is a locally finite irreducible infinite graph then

$$
h_{\text {top }}\left(\bar{X}_{E}, \bar{\sigma}_{E}\right)=h_{\text {top }}\left(\bar{\Sigma}_{E}, \bar{\sigma}_{E}\right) .
$$

Proof: Consider the open cover $\mathcal{P}_{n}:=\{[1], \ldots,[1 / n],[\overline{1 / n}]\}$ of $\bar{\Sigma}_{E}$, where

$$
\begin{aligned}
& {[1 / k]=\left\{\left(x_{i}\right) \in \bar{\Sigma}_{E} \mid x_{1}=1 / k\right\}, k=1, \ldots, n,} \\
& {[\overline{1 / n}]=\left\{\left(x_{i}\right) \in \bar{\Sigma}_{E} \mid x_{1}<1 / n\right\} .}
\end{aligned}
$$

Put $\mathcal{V}_{n}:=\bar{\sigma}_{E}^{n} \mathcal{P}_{n} \vee \bar{\sigma}_{E}^{n-1} \mathcal{P}_{n} \vee \cdots \vee \bar{\sigma}_{E} \mathcal{P}_{n} \vee \mathcal{P}_{n} \vee \bar{\sigma}_{E}^{-1} \mathcal{P}_{n} \vee \cdots \vee \bar{\sigma}_{E}^{n} \mathcal{P}_{n}$. Then

$$
\begin{aligned}
h_{\mathrm{top}}\left(\bar{\sigma}_{E},\right. & \left.\mathcal{V}_{n}\right) \\
& =\lim _{k \rightarrow \infty} \frac{1}{k} \log N\left(\bigvee_{l=0}^{k-1} \bar{\sigma}_{E}^{-l}\left(\mathcal{V}_{n}\right)\right) \\
& =\lim _{k \rightarrow \infty} \frac{1}{k} \log N\left(\bar{\sigma}_{E}^{n} \mathcal{P}_{n} \vee \cdots \vee \bar{\sigma}_{E}^{1} \mathcal{P}_{n} \vee \cdots \vee \mathcal{P}_{n} \vee \cdots \vee \bar{\sigma}_{E}^{n-k+1} \mathcal{P}_{n}\right) \\
& \leqslant \lim _{k \rightarrow \infty}\left(\frac{1}{k} \log N\left(\bar{\sigma}_{E}^{n} \mathcal{P}_{n} \vee \cdots \vee \bar{\sigma}_{E}^{1} \mathcal{P}_{n}\right)+\frac{1}{k} \log N\left(\mathcal{P}_{n} \vee \cdots \vee \bar{\sigma}_{E}^{-n-k+1} \mathcal{P}_{n}\right)\right) \\
& =\lim _{k \rightarrow \infty} \frac{1}{k} \log N\left(\mathcal{P}_{n} \vee \bar{\sigma}_{E}^{-1} \mathcal{P}_{n} \vee \cdots \vee \bar{\sigma}_{E}^{-n-k+1} \mathcal{P}_{n}\right) .
\end{aligned}
$$

Similarly for the finite open cover $\mathcal{Q}_{n}:=\{[1], \ldots,[1 / n],[\overline{1 / n}]\}$ of $\bar{X}_{E}$, where

$$
\begin{aligned}
& {[1 / k]=\left\{\left(x_{i}\right) \in \bar{X}_{E} \mid x_{1}=1 / k\right\}, k=1, \ldots, n,} \\
& {[\overline{1 / n}]=\left\{\left(x_{i}\right) \in \bar{X}_{E} \mid x_{1}<1 / n\right\},}
\end{aligned}
$$


and for $\mathcal{U}_{n}:=\mathcal{Q}_{n} \vee \bar{\sigma}_{E}^{-1} \mathcal{Q}_{n} \vee \cdots \vee \bar{\sigma}_{E}^{-n} \mathcal{Q}_{n}$, one has

$$
h_{\text {top }}\left(\bar{X}_{E}, \mathcal{U}_{n}\right)=\lim _{k \rightarrow \infty} \log N\left(\mathcal{Q}_{n} \vee \bar{\sigma}_{E}^{-1} \mathcal{Q}_{n} \vee \cdots \vee \bar{\sigma}_{E}^{-n-k+1} \mathcal{Q}_{n}\right)
$$

But $N\left(\mathcal{P}_{n} \vee \bar{\sigma}_{E}^{-1} \mathcal{P}_{n} \vee \cdots \vee \bar{\sigma}_{E}^{-n-k+1} \mathcal{P}_{n}\right)=N\left(\mathcal{Q}_{n} \vee \bar{\sigma}_{E}^{-1} \mathcal{Q}_{n} \vee \cdots \vee \bar{\sigma}_{E}^{-n-k+1} \mathcal{Q}_{n}\right)$ follows easily, thus from (4) and (5), we have

$$
h_{\text {top }}\left(\bar{\Sigma}_{E}, \mathcal{V}_{n}\right)=h_{\text {top }}\left(\bar{X}_{E}, \mathcal{U}_{n}\right)
$$

On the other hand, the sequence $\left\{\mathcal{U}_{n}\right\}\left(\left\{\mathcal{V}_{n}\right\}\right.$, respectively) is refining (see [1]), that is, $\mathcal{U}_{n+1}$ is a refinement of $\mathcal{U}_{n}$ and for every (finite) open cover $\mathcal{B}$ there exists an $n$ such that $\mathcal{U}_{n}$ is a refinement of $\mathcal{B}$, which implies that

$$
\begin{aligned}
& h_{\text {top }}\left(\bar{X}_{E}, \bar{\sigma}_{E}\right)=\lim _{n \rightarrow \infty} h_{\text {top }}\left(\bar{X}_{E}, \mathcal{U}_{n}\right) \\
& h_{\text {top }}\left(\bar{\Sigma}_{E}, \bar{\sigma}_{E}\right)=\lim _{n \rightarrow \infty} h_{\text {top }}\left(\bar{\Sigma}_{E}, \mathcal{V}_{n}\right)
\end{aligned}
$$

REMARK 4.3. For an infinite graph $E$, Gurevic [8] introduced an entropy

$$
\sup \left\{h\left(\Sigma_{E^{\prime}}\right) \mid E^{\prime} \subset E \text { finite subgraph }\right\}
$$

and proved that $h_{\text {top }}\left(\bar{\Sigma}_{E}\right)=\sup _{E^{\prime}} h\left(\Sigma_{E^{\prime}}\right)$ holds if $E$ is irreducible. Moreover the supremum can be taken over all the irreducible finite subgraphs by [8, Lemma 2].

THEOREM 4.4. Let $E$ be a locally finite irreducible infinite graph. Then

$$
h_{\mathrm{top}}\left(\bar{X}_{E}\right)=\sup _{E^{\prime}} h\left(X_{E^{\prime}}\right) \leqslant \operatorname{ht}\left(\phi_{E}\right)
$$

where the supremum is taken over all the finite subgraphs of $E$.

Proof: Recall that $h\left(\Sigma_{E^{\prime}}\right)=h\left(X_{E^{\prime}}\right)$ for any finite subgraph $E^{\prime}$ of $E$ (see Remark 2.1(b)). Then the first equality follows from Lemma 4.2 and Remark 4.3.

Note that for the locally compact shift space $X_{E}\left(\subset\left(\bar{E}^{1}\right)^{\mathrm{N}}\right)$ the cylinder sets

$$
[\alpha]=\left\{x=\left(x_{1}, x_{2}, \ldots\right) \in X_{E}\left|x_{i}=\alpha_{i}, 1 \leqslant i \leqslant\right| \alpha \mid\right\}, \alpha \in E^{*}
$$

are both compact and open and form a basis for the topology. Also one can easily show that the closure $\bar{X}_{E}$ is nothing but the one point compactification of $X_{E}$. As in a finite graph case, let

$$
\mathcal{D}_{E}:=C^{*}\left\{p_{\alpha} \mid \alpha \in E^{*}\right\}
$$

be the commutative $C^{*}$-subalgebra of $C^{*}(E)$ generated by projections $p_{\alpha}=s_{\alpha} s_{\alpha}^{*}$. Then clearly $\phi_{E}\left(\mathcal{D}_{E}\right) \subset \mathcal{D}_{E}$, hence $\mathrm{ht}\left(\left.\phi_{E}\right|_{\mathcal{D}_{E}}\right) \leqslant \mathrm{ht}\left(\phi_{E}\right)$. Thus it suffices to see that

$$
\operatorname{ht}\left(\left.\phi_{E}\right|_{\mathcal{D}_{E}}\right)=h_{\mathrm{top}}\left(\bar{X}_{E}\right) \text {. }
$$


We prove that the map $w: \mathcal{D}_{E} \rightarrow C_{0}\left(X_{E}\right), w\left(p_{\alpha}\right)=\chi_{[a]}$, is a $*$-isomorphism such that

$$
w\left(\left.\phi_{E}\right|_{D_{E}}\right) w^{-1}=\sigma_{E}^{*}
$$

which then implies that $h t\left(\left.\phi_{E}\right|_{\mathcal{D}_{E}}\right)=\operatorname{ht}\left(\sigma_{E}^{*}\right)$, and thus by Remark 2.4(b) we have

$$
\operatorname{ht}\left(\left.\phi_{E}\right|_{\mathcal{D}_{E}}\right)=\operatorname{ht}\left(\left.\tilde{\phi}_{E}\right|_{\tilde{\mathcal{D}}_{E}}\right)=h_{\mathrm{top}}\left(\bar{X}_{E}\right)
$$

Since it is tedious to show that $w$ is an injective $*$-homomorphism satisfying (6), here we only prove that $w$ is surjective. It is enough to see that the linear span of the characteristic functions $\chi_{[\alpha]}$ is dense in $C_{0}\left(X_{E}\right)$. Let $f \in C_{0}\left(X_{E}\right)$ and $\varepsilon>0$. Then there is a compact subset $K \subset X_{E}$ such that $\left\|\left.f\right|_{X_{E} \backslash K}\right\|<\varepsilon$. For each $x=\left(x_{n}\right) \in K$, consider the cylinder set

$$
[x]_{n}:=\left\{y=\left(y_{n}\right) \in X_{E} \mid x_{k}=y_{k}, 1 \leqslant k \leqslant n\right\} .
$$

Since $f$ is continuous at $x$ there is a neighborhood $U_{x}$ of $x$ such that

$$
|f(x)-f(y)|<\varepsilon \text { whenever } y \in U_{x} .
$$

Moreover we can choose $U_{x}=[x]_{N}$ for some $N \in \mathbb{N}$. Then there exists a finite subcover of $\left\{U_{x} \mid x \in K\right\}$ consisting of disjoint open sets, say $\left\{\left[x^{1}\right]_{N_{1}}, \ldots,\left[x^{m}\right]_{N_{m}}\right\}$. Put $g:=\sum_{j=1}^{m} f\left(x^{j}\right) \chi_{\left[x^{j}\right]_{N_{j}}}$. Then $g(y)=0$ for $y \notin \bigcup_{j=1}^{m}\left[x^{j}\right]_{N_{j}}$. If $y \in\left[x^{j}\right]_{N_{j}}$ for some $j$ then

$$
|f(y)-g(y)| \leqslant\left|f(y)-f\left(x^{j}\right)\right|+\left|f\left(x^{j}\right)-g(y)\right|<\varepsilon .
$$

Therefore $|g(y)-f(y)|<\varepsilon$ for each $y \in X_{E}$.

REMARK 4.5. It would be nice to obtain an upper bound for the topological entropy ht $\left(\phi_{E}\right)$ for $E$ in Theorem 4.4. Let $E$ be a locally finite irreducible infinite graph and let $\mathcal{A}_{E}$ be the AF subalgebra of $C^{*}(E)=C^{*}\left\{p_{v}, s_{e}\right\}$ generated by the partial isometries of the form $s_{\alpha} s_{\beta}^{*}$ with $|\alpha|=|\beta|$. Then $\mathcal{A}_{E}$ is $\phi_{E}$-invariant and contains the commutative subalgebra $\mathcal{D}_{E}$, so that $\operatorname{ht}\left(\left.\phi_{E}\right|_{\mathcal{D}_{E}}\right) \leqslant \operatorname{ht}\left(\left.\phi_{E}\right|_{\mathcal{A}_{E}}\right)$. We shall give an upper bound for $\operatorname{ht}\left(\left.\phi_{E}\right|_{\mathcal{A}_{E}}\right)$ elsewhere.

\section{REFERENCES}

(1] R.L. Adler, A.G. Konheim and M.H. McAndrew, 'Zoological entropy', Trans. Amer. Math. Soc. 114 (1965), 309-319.

[2] T. Bates, J.H. Hong, I. Raeburn and W. Szymanski, 'The ideal structure of the $C^{*}$-algebras of infinite graphs', Illinois J. Math. 46 (2002), 1159-1176.

[3] F.P. Boca and P. Goldstein, 'Topological entropy for the canonical endomorphism of Cuntz-Krieger algebras', Bull. London Math. Soc. 32 (2000), 345-352.

[4] N.P. Brown, 'Topological entropy in exact $C^{*}$-algebras', Math. Annalen 314 (1999), 347-367. 
[5] M. Choda, 'Endomorphisms of shift type (entropy for endomorphisms of Cuntz algebras)', in Operator Algebras and Quantum Field Theory (Rome, 1996) (International Press, Cambridge, MA), pp. 469-475.

[6] J. Cuntz and W. Krieger, 'A class of $C^{*}$-algebras and topological Markov chains', Invent. Math. 56 (1980), 251-268.

[7] V. Deaconu, 'Entropy estimates for some $C^{*}$-endomorphisms', Proc. Amer. Math. Soc. 127 (1999), 3653-3658.

[8] B.M. Gurevic, 'Topological entropy of enumerable Markov chains', (in Russian), Dokl. Akad. Nauk SSSR 187 (1969), 216-226.

[9] E. Kirchberg, 'On subalgebras of the CAR-algebra', J. Funct. Anal. 129 (1995), 35-63.

[10] B.P. Kitchens, Symbolic dynamics (Springer-Verlag, Berlin, Heidelberg, New York, 1998).

[11] A. Kumjian, 'Notes on $C^{*}$-algebras of graphs', Contemp. Math. 228 (1998), 189-200.

[12] A. Kumjian, D. Pask and I. Raeburn, 'Cuntz-Krieger algebras of directed graphs', Pacific J. Math. 184 (1998), 161-174.

[13] D. Lind and B. Marcus, An introduction to symbolic dynamics and coding (Cambridge University Press, Cambridge, 1999).

[14] C. Pinzari, Y. Watatani and K. Yonetani, 'KMS states, entropy and the variational principle in full $C^{*}$-dynamical systems', Comm. Math. Phys. 213 (2000), 331-379.

[15] D. Voiculescu, 'Dynamical approximation entropies and topological entropy in operator algebras', Comm. Math. Phys. 170 (1995), 249-281.

[16] P. Walters, An introduction to ergodic theory, Graduate Texts in Mathematics 79 (Springer-Verlag, publaddr Berlin, Heidelberg, New York 1982).

Mathematical Sciences Division BK21, Seoul National University

Seoul, 151-742

Korea

e-mail: jajeong@math.snu.ac.kr
Department of Mathematics

Hanshin University

Osan, 447-791

Korea

e-mail: ghpark@hanshin.ac.kr 